\title{
Diversity and Abundance of Birds in Agricultural Zone Associated to the Bay Santa María La Reforma, Sinaloa, Mexico
}

\section{Guadalupe Humberto Gurrola López ${ }^{1,2,3}$, Nicolás Castañeda Lomas ${ }^{1}$, Laura Beatriz Rivera Rodríguez ${ }^{1}$, Leopoldo Partida Ruvalcaba ${ }^{2 *}$, Feliciano J. Heredia Pineda ${ }^{4}$}

\footnotetext{
${ }^{1}$ Posgrado en Ciencias en Recursos Acuáticos, Facultad de Ciencias del Mar, Universidad Autónoma de Sinaloa, Mazatlán, México ${ }^{2}$ Universidad Tecnológica de Culiacán, Culiacán de Rosales, México

${ }^{3}$ Escuela de Biología, Universidad Autónoma de Sinaloa, Culiacán de Rosales, México

${ }^{4}$ Laboratorio de Fauna Silvestre, Departamento de Recursos Naturales Renovables, Universidad Autónoma Agraria Antonio Narro, Saltillo, México

Email: gh_gurrola@ hotmail.com, *parpolo@yahoo.com.mx
}

How to cite this paper: Gurrola López, G.H. Castañeda Lomas, N., Rodríguez, L.B.R., Ruvalcaba, L.P. and Pineda, F.J.H. (2016) Diversity and Abundance of Birds in Agricultural Zone Associated to the Bay Santa María La Reforma, Sinaloa, Mexico. Open Access Library Journal, 3: e3229.

http://dx.doi.org/10.4236/oalib.1103229

Received: November 14, 2016

Accepted: December 2, 2016

Published: December 5, 2016

Copyright $\odot 2016$ by authors and Open Access Library Inc.

This work is licensed under the Creative Commons Attribution International

License (CC BY 4.0).

http://creativecommons.org/licenses/by/4.0/

(c) (i) Open Access

\section{Abstract}

The objective of this research was to determine bird's abundance and diversity associated in an agricultural system at the Bahia Santa Maria La Reforma (BSM-LR), which may represent a risk to crops and/or have a value for conservation. Annual monthly samplings were conducted to determine the richness and abundance of the birds; in seven linear transects, distributed throughout the agricultural area, all birds seen and heard were recorded. Bird species were identified using the field guides for birds in Mexico and North America and binoculars $(8 \times 40)$. Richness was determined from the accumulation of species seen over the sampling time, and the specific abundance with the sum of the number of individuals seen each month. The Alpha diversity was determined using the Shannon Wiener index and the monthly abundance data for each month sampled. A total of 15,427 birds from 110 species were found. This diversity is grouped into 16 orders, 37 families and 90 genera, of which Agaleus phoeiceus, Quiscalus mexicanus, Zenaida macroura and Columbina passerina were the most abundant, accounting for $77.6 \%$ of the total Birds seen; the exotic species found were Streptopelia decaocto, Sturnus vulgaris and Passer domesticus, and 16 species are of conservation concern.

\section{Subject Areas}

Agricultural Science 


\section{Keywords}

Birdlife, Agriculture, Gulf of California, Agro-Ecology, Species of Conservation Concern

\section{Introduction}

El desarrollo agrícola en Sinaloa, detonó entre 1970 y 1990 al duplicarse la superficie de irrigación, principalmente en terrenos ejidales, aumentando su aportación económica y su impacto al ambiente en cuanto al recurso agua y el suelo se refiere [1]. En Sinaloa el impacto ocasionado en la distribución y abundancia de poblaciones silvestres debido a la transformación de hábitat natural a cultivos agrícolas ha sido poco evaluado. En este sentido, la facilidad de observar aves y que las poblaciones de muchas especies han disminuido, facilita el que sean utilizados para evaluar el impacto de cambios de hábitat [2].

La agricultura es la actividad primaria de mayor importancia para la producción de alimentos en el mundo [3] y su crecimiento está ligado a la pérdida de cobertura vegetal [4], por lo que es necesario evaluar si la perdida y/o fragmentación de hábitat se considera una de las principales causas del decremento de poblaciones de aves silvestres [5] y, por lo tanto, la consecuente disminución de la biodiversidad [6] y ser causal de que las especies se encuentren amenazadas [7]. En este sentido, generar datos sobre la diversidad biológica se convierte en un elemento básico que permite planificar las políticas de conservación a una escala adecuada [2] [8] [9]; sin embargo, en México no sólo se afronta el problema de la pérdida de la biodiversidad, sino también el de la existencia de vacíos de información sobre el conocimiento de la misma [10] [11].

La biodiversidad es importante por la variedad de servicios ambientales que brinda [11] [12] [13]. Mantener y conservar esa diversidad biológica es un objetivo de la humanidad para conservar la riqueza natural en el planeta y hacer sustentable toda acción en su racional aprovechamiento, incluyendo la agricultura [14]. Para el caso de las aves, Navarro y colaboradores [15] señalan que se han descrito entre 1123 y 1150 especies en México y considera que este grupo taxonómico está bien conocido. De estas se reportan 486 para Sinaloa [16] [17]. Sin embargo, poco se sabe sobre las interacciones que se dan entre ellas y la actividad agrícola.

Este trabajo contribuye a generar conocimiento sobre la abundancia y diversidad ornitológica de la zona agrícola asociada a la BSM-LR, ya que consideramos que las nuevas condiciones ambientales de los sistemas agrícolas representan un hábitat importante para el desarrollo de especies de interés para la conservación así como para especies oportunistas que por su abundancia pueden afectar económicamente la actividad. Esto, con el fin de que esta información sea tomada como antecedente técnico para considerar estos ecosistemas de origen antropogénico en el diseño de esquemas de conservación como las ANP ya que usualmente son excluidos por considerarse sistemas transformados para el beneficio del hombre. 


\section{Materiales y Métodos}

La BSM-LR es un humedal costero con un área húmeda de $586 \mathrm{~km}^{2}$, ubicada en la zona centro-norte del estado de Sinaloa (Figure 1), y se une al Golfo de California en su parte sur por la boca Punta Yameto de $3.4 \mathrm{~km}$ de amplitud y al norte por la boca de Punta del Perihuete con $5.3 \mathrm{~km}$ de abertura, está protegida del mar abierto por la Isla Altamura que forma una barrera arenosa de $42 \mathrm{~km}$ que separa ambas bocas, su profundidad promedio es de $7 \mathrm{~m}$ y alcanza una máxima de $24 \mathrm{~m}$ [18] [19]. La presencia de diferentes tipos de vegetación y grandes planicies lodosas e inundables, le confieren diferentes hábitat que son utilizados por altas concentraciones de aves de diferentes especies [20], por lo que es considerada, a nivel nacional e internacional, como un sitio de importancia para la conservación [21]. Entre las actividades económicas que se desarrollan en la bahía son la agricultura, acuicultura, cacería deportiva y explotación de sal [22]. El crecimiento de la zona agrícola de BSM-LR alcanzó 33,295 ha en 1992, lo que representa un incremento de $119.8 \%$ de su cobertura inicial con respecto a las 15,145 existentes en 1976, a una tasa de crecimiento anual de 955.3 ha [4], los principales cultivos son maíz, frijol, sorgo y garbanzo.

Para el presente trabajo se realizaron monitoreos mensuales durante el año en siete transectos lineales, distribuidos en toda la zona agrícola de BSM-LR, en los que se registraron todas las aves vistas y escuchadas [6]. Los conteos se realizaron por la mañana, durante las primeras 4.5 horas posteriores a la salida de sol, mientras que por la tarde se iniciaron tres horas antes de la postura del sol al presentar mayor actividad [23] [24].

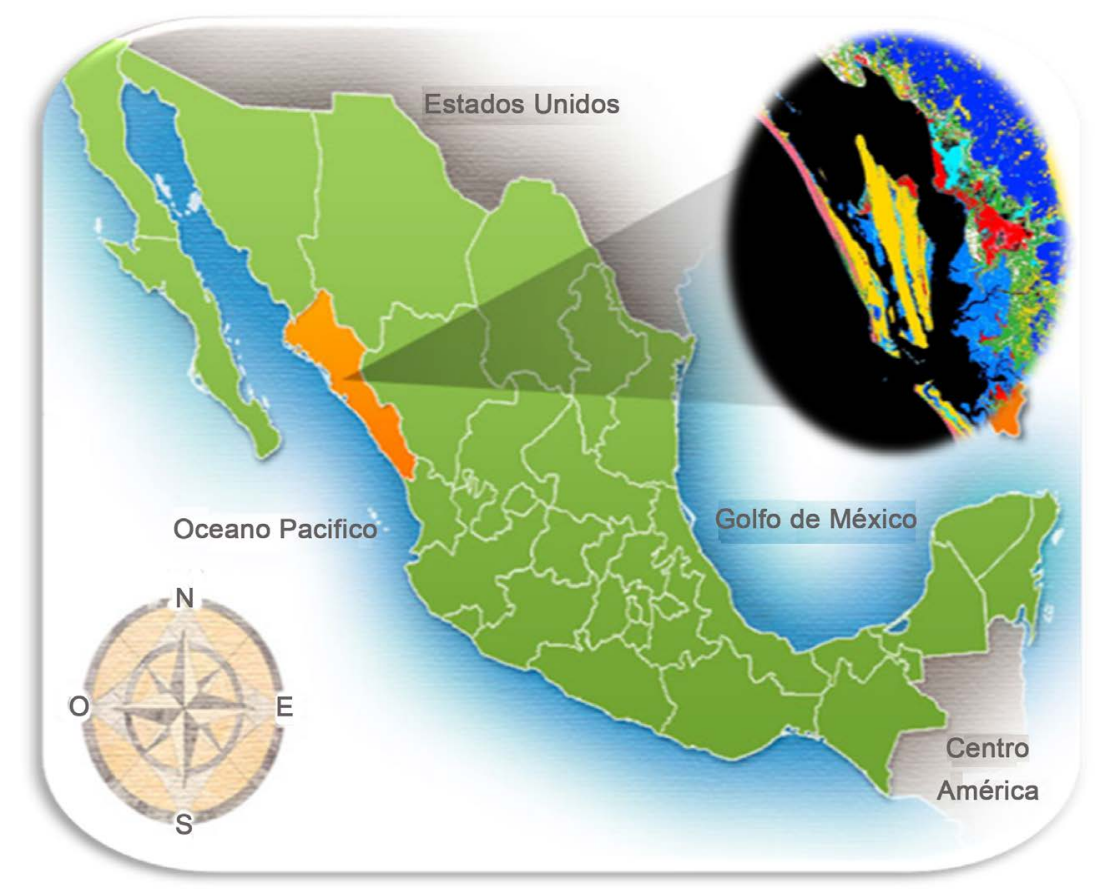

Figure 1. Ubicación de Bahía Santa María La Reforma en la zona centro-norte del Estado de Sinaloa, México. 
La identificación de las especies se realizó mediante guías de campo para las aves de México y Norteamérica [25] [26] [27] [28] y el uso de binoculares $(8 \times 40)$. Las especies se contaron directamente cuando se trató de parvadas menores a 300 individuos [29] [30], en parvadas mayores a 300 fue estimada utilizando el método de bloques que se basa en el conteo de una porción de la parvada, para posteriormente estandarizarlas con el resto [31] [32]. Se utilizó un vehículo automotor que se desplazaba a una velocidad máxima de $20 \mathrm{~km}$ por hora, que permite llevar a cabo la identificación y conteo de las aves [6]. Cuando se requirió estimar o identificar especies escurridizas o silenciosas se detuvo completamente el vehículo [23]. La lista de aves se integró siguiendo la taxonomía adoptada por la American Ornithologists' Union [33] en la séptima versión de su lista de aves de Norte y Centro América, que incluye los cambios hasta el suplemento 56 [34].

Se consideraron como especies de interés para la conservación a las que están incluidas en la NOM-059 SEMARNAT-2010 [35] y en la Unión Internacional para la Conservación de la Naturaleza [36], así como aquéllas que representan algún grado de endemismo para México [37].

La riqueza se obtuvo de la acumulación de las especies vistas a lo largo del tiempo de muestreos [38] y la abundancia especifica de la sumatoria del número de individuos vistos cada mes. Mediante el índice de Shannon Wiener [39] y los datos de abundancia mensual se calculó la diversidad alfa para cada mes muestreado en la zona agrícola.

\section{Resultados y Discusión}

Diversidad y abundancia. Se observaron un total de 15,427 aves de 110 especies, esta diversidad se agrupa en 16 órdenes, 37 familias y 90 géneros (Table 1). El orden Passeriformes fue el más representado con 16 familias y 46 especies, seguido de Charadriforme con 4 familias y 13 especies; ambos representan el $53.6 \%$ de las especies que integran el listado; las familias con mayor diversidad de especies se muestran en la Figure 2. Los meses con mayor riqueza fueron diciembre, febrero y abril con 51

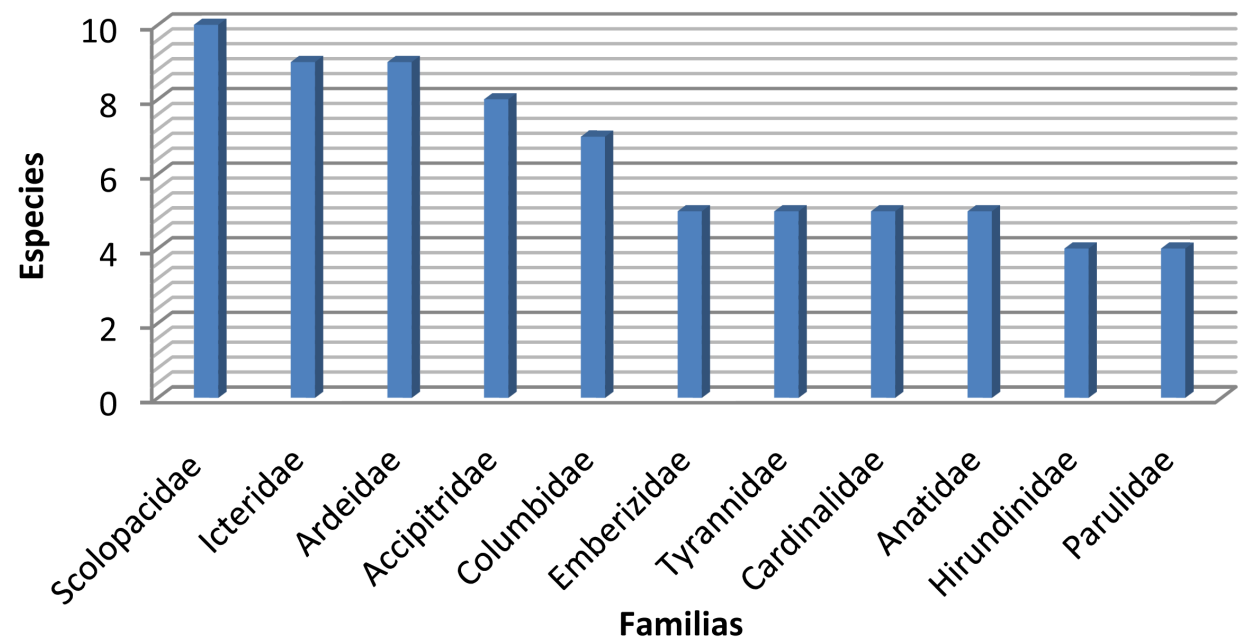

Figure 2. Familias con mayor diversidad de especies. 
Table 1. Lista de especies observadas en la zona agrícola de Bahía Santa María La Reforma, Sinaloa, México.

\begin{tabular}{|c|c|c|c|c|c|c|c|}
\hline No. & Orden & Familia & Especie & NOM & G E & EDR & Residencia \\
\hline 1 & Anseriformes & Anatidae & Dendrocygna autumnalis & & & & $\mathrm{R}$ \\
\hline 2 & & & Anas platyrhynchos & & & & I \\
\hline 3 & & & Anas discors & & & & I \\
\hline 4 & & & Anas clypeata & & & & I \\
\hline 5 & & & Aythya affinis & & & & I \\
\hline 6 & Galliformes & Odontophoridae & Callipepla duoglasit ${ }^{21}$ & & $\mathrm{E}$ & * & $\mathrm{R}$ \\
\hline 7 & & & Callipepla gambelii & & & & $\mathrm{R}$ \\
\hline 8 & Ciconiiformes & Ciconiidae & Mycteria americana ${ }^{1}$ & $\mathrm{PR}$ & & & $\mathrm{R}$ \\
\hline 9 & Suliformes & Phalacrocoracidae & Phalacrocorax brasilianus & & & & $\mathrm{R}$ \\
\hline 10 & Pelecaniformes & Ardeidae & Botaurus lentiginosus $^{1}$ & A & & & I \\
\hline 11 & & & Ardea herodias & & & & $\mathrm{R}$ \\
\hline 12 & & & Ardea alba & & & & $\mathrm{R}$ \\
\hline 13 & & & Egretta thula & & & & $\mathrm{R}$ \\
\hline 14 & & & Egretta caerulea & & & & $\mathrm{R}$ \\
\hline 15 & & & Egretta tricolor & & & & $\mathrm{R}$ \\
\hline 16 & & & Bubulcus ibis & & & & $\mathrm{R}$ \\
\hline 17 & & & Butorides virescens & & & & $\mathrm{R}$ \\
\hline 18 & & & Nycticorax nycticorax & & & & $\mathrm{R}$ \\
\hline 19 & & Threskiornithidae & Eudocimus albus & & & & $\mathrm{R}$ \\
\hline 20 & & & Plegadis chií & & & & I \\
\hline 21 & & & Platalea ajaja & & & & $\mathrm{R}$ \\
\hline 22 & Accipitriformes & Cathartidae & Coragyps atratus & & & & $\mathrm{R}$ \\
\hline 23 & & & Cathartes aura & & & & $\mathrm{R}$ \\
\hline 24 & & Pandionidae & Pandion haliaetus & & & & $\mathrm{R}$ \\
\hline 25 & & Accipitridae & Elanus leucurus & & & & $\mathrm{R}$ \\
\hline 26 & & & Accipiter striatus ${ }^{9}$ & $\mathrm{PR}$ & & & I \\
\hline 27 & & & Accipiter cooperii ${ }^{6}$ & $\mathrm{PR}$ & & & I \\
\hline 28 & & & Buteogallus anthracinus ${ }^{1}$ & $\mathrm{PR}$ & & & $\mathrm{R}$ \\
\hline 29 & & & Parabuteo unicinctus ${ }^{2}$ & $\mathrm{PR}$ & & & $\mathrm{R}$ \\
\hline 30 & & & Buteo nitidus & & & & $\mathrm{R}$ \\
\hline 31 & & & Buteo swainsont ${ }^{2}$ & PR & & & $\mathrm{T}$ \\
\hline 32 & & & Buteo jamaicensis & & & & I \\
\hline 33 & Charadriformes & Recurvirostridae & Himantopus mexicanus & & & & $\mathrm{R}$ \\
\hline 34 & & & Recurvirostra americana & & & & I \\
\hline 35 & & Charadriidae & Charadrius vociferus & & & & $\mathrm{R}$ \\
\hline
\end{tabular}




\section{Continued}

\begin{tabular}{|c|c|c|c|c|c|c|}
\hline 36 & & Scolopacidae & Actitis macularius & & & I \\
\hline 37 & & & Tringa melanoleuca & & & I \\
\hline 38 & & & Tringa semipalmata & & & I \\
\hline 39 & & & Tringa flavipes & & & I \\
\hline 40 & & & Numenius phaeopus & & & I \\
\hline 41 & & & Numenius americanus & & & I \\
\hline 42 & & & Calidris minutilla & & & I \\
\hline 43 & & & Limnodromus sp & & & I \\
\hline 44 & & Laridae & Leucophaeus atricilla & & & VNR \\
\hline 45 & & & Larus delawarensis & & & I \\
\hline 46 & Columbiformes & Columbidae & Patagioenas flavirostris & & & $\mathrm{R}$ \\
\hline 47 & & & Streptopelia decaocto & & & $\mathrm{R}$ \\
\hline 48 & & & Columbina inca & & & $\mathrm{R}$ \\
\hline 49 & & & Columbina passerina & & & $\mathrm{R}$ \\
\hline 50 & & & Columbina talpacoti & & & $\mathrm{R}$ \\
\hline 51 & & & Zenaida asiatica & & & $\mathrm{R}$ \\
\hline 52 & & & Zenaida macroura & & & I \\
\hline 53 & Cuculiformes & Cuculidae & Geococcyx californianus & & & $\mathrm{R}$ \\
\hline 54 & & & Crotophaga sulcirostris & & & $\mathrm{R}$ \\
\hline 55 & Strigiformes & Strigidae & Athene cunicularia & & & I \\
\hline 56 & Caprimulgiformes & Caprimulgidae & Chordeiles acutipennis & & & $\mathrm{R}$ \\
\hline 57 & Apodiformes & Trochilidae & Cynanthus latirostris ${ }^{13}$ & SE & & $\mathrm{R}$ \\
\hline 58 & & & Amazilia violiceps $^{1}$ & SE & & $\mathrm{R}$ \\
\hline 59 & Coraciiformes & Alcedinidae & Megaceryle alcyon & & & I \\
\hline 60 & & & Chloroceryle americana & & & $\mathrm{R}$ \\
\hline 61 & Piciformes & Picidae & Melanerpes uropygialis & & & $\mathrm{R}$ \\
\hline 62 & & & Picoides scalaris & & & $\mathrm{R}$ \\
\hline 63 & Falconiformes & Falconidae & Caracara cheriway & & & $\mathrm{R}$ \\
\hline 64 & & & Falco sparverius & & & I \\
\hline 65 & Passeriformes & Tyrannidae & Sayornis nigricans & & & I \\
\hline 66 & & & Pyrocephalus rubinus & & & $\mathrm{R}$ \\
\hline 67 & & & Pitangus sulphuratus & & & $\mathrm{R}$ \\
\hline 68 & & & Myiozetetes similis & & & $\mathrm{R}$ \\
\hline 69 & & & Tyrannus melancholicus & & & $\mathrm{R}$ \\
\hline 70 & & Laniidae & Lanius ludovicianus & & & $\mathrm{R}$ \\
\hline 71 & & Corvidae & Corvus sinaloae ${ }^{87}$ & $\mathrm{E}$ & * & $\mathrm{R}$ \\
\hline 72 & & Hirundinidae & Progne subis & & & $\mathrm{T}$ \\
\hline 73 & & & Tachycineta albilinea & & & $\mathrm{R}$ \\
\hline 74 & & & Stelgidopteryx serripennis & & & $\mathrm{R}$ \\
\hline 75 & & & Petrochelidon pyrrhonota & & & $\mathrm{T}$ \\
\hline 76 & & Remizidae & Auriparus flaviceps & & & $\mathrm{R}$ \\
\hline 77 & & Troglodytidae & $\begin{array}{l}\text { Campylorhynchus } \\
\text { brunneicapillus }\end{array}$ & & & $\mathrm{R}$ \\
\hline
\end{tabular}




\section{Continued}

\begin{tabular}{|c|c|c|c|c|}
\hline 78 & & Pheugopedius felix ${ }^{7}$ & $\mathrm{E}$ & $\mathrm{R}$ \\
\hline 79 & Polioptilidae & Polioptila nigriceps $s^{13}$ & $\mathrm{E}$ & $\mathrm{R}$ \\
\hline 80 & Mimidae & Toxostoma curvirostre & & $\mathrm{R}$ \\
\hline 81 & & Mimus polyglottos & & $\mathrm{R}$ \\
\hline 82 & Sturnidae & Sturnus vulgaris & & $\mathrm{R}$ \\
\hline 83 & Parulidae & Oreothlypis celata & & I \\
\hline 84 & & Geothlypis trichas & & $\mathrm{R}$ \\
\hline 85 & & Setophaga coronata & & I \\
\hline 86 & & Icteria virens & & $\mathrm{T}, \mathrm{I}$ \\
\hline 87 & Thraupidae & Sporophila torqueola & & $\mathrm{R}$ \\
\hline 88 & & Saltator coerulescens & & $\mathrm{R}$ \\
\hline 89 & Emberizidae & Peucaea carpalis ${ }^{18}$ & $\mathrm{CE}$ & $\mathrm{R}$ \\
\hline 90 & & Spizella passerina & & I \\
\hline 91 & & Chondestes grammacus & & I \\
\hline 92 & & Melospiza lincolnii & & I \\
\hline 93 & & Zonotrichia leucophrys & & I \\
\hline 94 & Cardinalidae & Cardinalis cardinalis & & $\mathrm{R}$ \\
\hline 95 & & Cardinalis sinuatus & & $\mathrm{R}$ \\
\hline 96 & & Cyanocompsa parellina & & $\mathrm{R}$ \\
\hline 97 & & Passerina amoen $a^{2}$ & SE & I \\
\hline 98 & & Passerina versicolor & & $\mathrm{R}$ \\
\hline 99 & Icteridae & Agelaius phoeniceus & & I \\
\hline 100 & & Sturnella neglecta & & I \\
\hline 101 & & $\begin{array}{l}\text { Xanthocephalus } \\
\text { xanthocephalus }\end{array}$ & & I \\
\hline 102 & & Quiscalus mexicanus & & $\mathrm{R}$ \\
\hline 103 & & Molothrus aeneus & & $\mathrm{R}$ \\
\hline 104 & & Molothrus ater & & $\mathrm{R}$ \\
\hline 105 & & Icterus spurius & & $\mathrm{T}$ \\
\hline 106 & & Icterus cucullatus $^{3}$ & SE & I \\
\hline 107 & & Icterus pustulatus & & $\mathrm{R}$ \\
\hline 108 & Fringillidae & Haemorhous mexicanus & & $\mathrm{R}$ \\
\hline 109 & & Spinus psaltria & & $\mathrm{R}$ \\
\hline 110 & Passeridae & Passer domesticus & & $\mathrm{R}$ \\
\hline
\end{tabular}

$\mathrm{NOM}=$ NOM 059 SEMARNAT 2010, $\mathrm{PR}=$ Protección Especial, $\mathrm{A}=$ Amenazada, $\mathrm{GE}=$ Grado de Endemismo, $\mathrm{E}=$ Endémicas, $\mathrm{SE}=$ Semiendémicas, $\mathrm{CE}=$ Cuasiendémicas, $\operatorname{EDR}\left({ }^{*}\right)=$ Especies con Distribución Restringida a Sinaloa. La estacionalidad se asignó de acuerdo con los criterios de Howell y Webb. 1995. $\mathrm{R}$ = Residentes Reproductor, $\mathrm{I}=$ Visitante invernal, $\mathrm{VNR}=$ Visitante no reproductivo, $\mathrm{RV}=$ Residente de verano $\mathrm{T}=$ Migrante Transitorio. 
especies, mientras que los meses con mayor abundancia fueron abril, mayo y junio (Figure 3), la presencia de sólo 30 especies en el mes de septiembre se puede explicar por la presencia del huracán Manuel que, el 19 de septiembre de 2013, impactó la costa del centro de Sinaloa, una semana antes del muestreo.

La riqueza de especies observadas en la zona agrícola representó el $35.5 \%$ de las especies que reporta Comisión Nacional de Áreas Naturales Protegidas para la BSM-LR [40], de las cuales por su condición exótica sobresalieron Streptopelia decaocto, Sturnus vulgaris y Passer domesticus, que generalmente se observan en ambientes urbanos, pero que ahora amplían su distribución a zonas agrícolas, donde en el futuro pueden representar un peligro para la producción agrícola, como sucede con otras especies plaga que reporta Martínez-López [41].

Las zonas agrícolas son ambientes modificados por el hombre para la producción de alimento, cambios que generan condiciones ambientales que favorecen a ciertas especies granívoras, que por la alta disponibilidad de alimento tienden a incrementar sus poblaciones [2] [42]. En la zona agrícola de BSM-LR, las especies más abundantes fueron Agelaius phoeniceus, Quiscalus mexicanus, Zenaida macroura y Columbina passerina, con el $77.6 \%$ del total de las aves presentes, su abundancia puede representar un peligro para la producción de granos, ya que se ha observado que las poblaciones aumentan a partir de abril hasta agosto, cuando los cultivos producen espigas o mazorcas y el ciclo agrícola termina (Figure 3). Estos resultados concuerdan con Martínez López [41], quien reporta a Quiscalus mexicanus Agelaius phoeniceus y Molothrus ater como especies que ocasionan pérdidas económicas en la agricultura sinaloense.

Especies de interés para la conservación. De las 110 especies vistas en la zona agrícola de BSM-LR, 16 (14.6\%) son de interés para la conservación (Table 1) de las cuales siete están bajo estatus de protección, de acuerdo a la NOM-059 SEMARNAT 2010 (una amenazada y seis en protección especial), nueve presentan algún endemismo para México (cuatro endémicas, cuatro semi-endémicas y una cuasi-endémica) y dos presentan distribución restringida para Sinaloa. Las familias con mayor número de

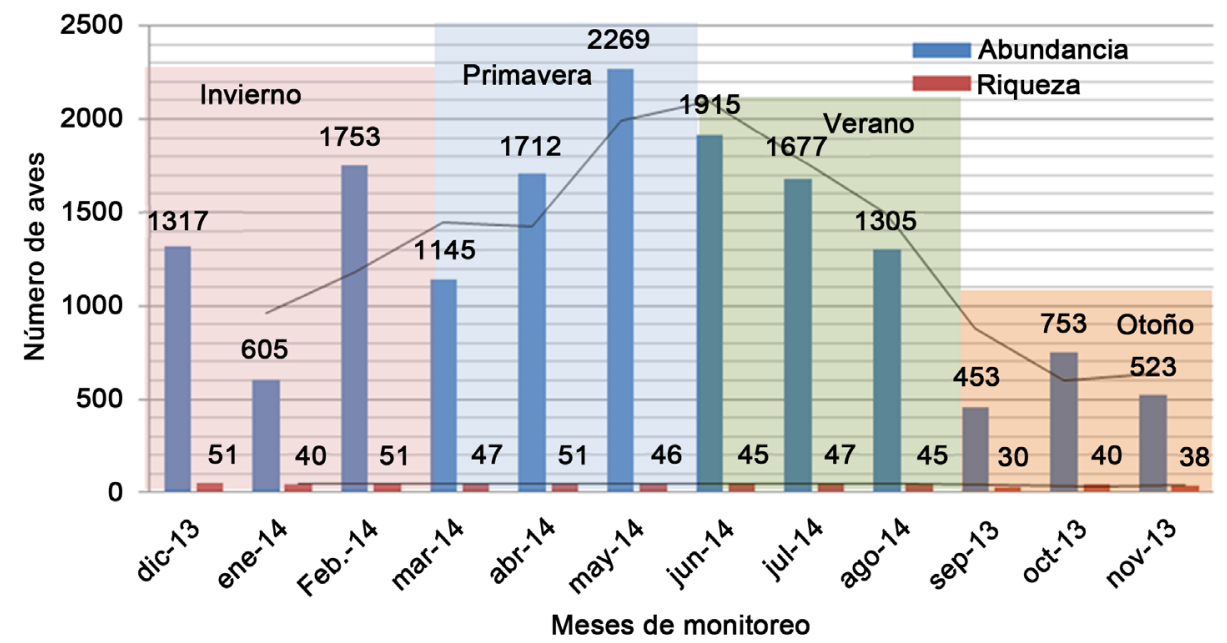

Figure 3. Riqueza y abundancia de aves presentes en la zona agrícola de BSM-LR. 
especies de interés para la conservación son accipitridae con cinco y emberizidae con tres. Las especies de interés para la conservación son las que menor abundancia tuvieron en la zona agrícola (Table 1), quizás porque su presencia en el sistema agrícola se relaciona con los nichos ecológicos que resultaron debido a la transformación de hábitat primarios en zonas agrícolas.

$\mathrm{Al}$ respecto, Rodríguez-Estrella [2] señala que la fragmentación y transformación de ecosistemas desérticos a campos agrícolas y urbanos han afectado la distribución y abundancia de seis especies altamente selectivas al matorral desértico de Baja California; asimismo, que las actividades humanas pueden jugar un papel importante en la conservación de las especies. En tanto que para Sinaloa, Gurrola [4] evaluó cambios en la cobertura vegetal y uso de suelo de BSM-LR en el periodo de 1973 a 1992 (19 años), mediante el uso de la técnica de percepción remota, y señala que el crecimiento del área agrícola asociada a la bahía ocupa áreas que originalmente estaban cubiertas por bosque espinoso y vegetación halofita, y que estos cambios pudieron afectar a las poblaciones de aves locales y migratorias.

En este trabajo se presentan datos sobre diversidad de especies en la zona agrícola asociada a BSM-LR; sin embargo, no se cuenta con datos históricos para saber qué tipo de impacto ha tenido la transformación de bosque espinoso y vegetación halofita en la abundancia y distribución de las poblaciones de aves.

La diversidad de especies de aves anual para la zona agrícola asociada a BSM-LR fue de 2.7 relativamente bajo con respecto a 4.70 que se esperaría si todas las especies tuvieran la misma abundancia [39]. La disparidad en la abundancia de las especies que componen la comunidad de aves, indica que existe poca uniformidad de los valores de importancia de las especies en el sistema agrícola y, por lo tanto, indica la presencia de especies con alta representatividad y especies raras con presencia de muy pocos representantes. En la Table 2 se observan los datos de abundancia, riqueza específica y

Table 2. Datos de abundancia, riqueza de especies y del índice de Shannon Wiener, a lo largo del periodo de muestreo.

\begin{tabular}{cccc}
\hline Mes & Abundancia & Riqueza especifica & Índice de Shannon Wiener \\
\hline Diciembre & 1317 & 51 & 2.08 \\
Enero & 605 & 40 & 2.44 \\
Febrero & 1753 & 51 & 2.37 \\
Marzo & 1145 & 47 & 2.77 \\
Abril & 1712 & 51 & 2.41 \\
Mayo & 2269 & 46 & 1.99 \\
Junio & 1915 & 45 & 2.23 \\
Julio & 1677 & 47 & 2.66 \\
Agosto & 1305 & 45 & 2.52 \\
Septiembre & 453 & 30 & 2.45 \\
Octubre & 753 & 40 & 2.66 \\
Noviembre & 523 & 38 & 2.47 \\
Total anual & 15427 & 110 & 2.70 \\
\hline
\end{tabular}


del índice de Shannon Wiener, obtenidos para cada uno de los meses que incluyó el muestreo. Esta disparidad en abundancia de las especies coincide con los resultados de Carrascal y Palomino [43] sobre las elevadas densidades y riqueza de especies encontradas en las zonas agrícolas de la Isla Tenerife del archipiélago canario, localizada a $288 \mathrm{~m}$ de la costa Africana.

\section{Conclusiones}

La diversidad y riqueza de aves en la Bahía Santa María La Reforma fue del tamaño de 110 especies; mientras que la abundancia se puso de manifiesto con más de 15,000 aves presentes, con predominancia de las especies Agelaius phoeniceus, Quiscalus mexicanus, Zenaida macroura y Columbina passerina, que junto con las exóticas Streptopelia decaocto, Sturnus vulgaris y Passer domesticus pueden representar un peligro para las zonas agrícolas, específicamente para los cultivos de maíz y sorgo que se siembran en la zona agrícola asociada a dicha bahía, lo que quizás influye para que esta área sea un hábitat muy importante para las especies presentes.

\section{References}

[1] Norzagaray-Campos, M., García-Gutiérrez, C., Llanes-Cárdenas, O., Troyo-Diéguez, E. and Muñoz-Sevilla, P. (2010) Análisis de la producción agrícola extensiva en Sinaloa: Alternativas para el uso sostenible del agua. Ra Ximhai, 6, 45-50.

[2] Rodríguez, E.R. (2007) Land Use Changes Affect Distributional Patterns of Desert Birds in the Baja California Peninsula, Mexico. Diversity and Distribution, 13, 877-889. https://doi.org/10.1111/j.1472-4642.2007.00387.x

[3] Sauceda-López, R. and Gómez-Soto, M.P. (2003) La agricultura y sus efectos medioambientales. In: Cifuentes, L.J. and Gaxiola-López, J., Eds., Atlas de los Ecosistemas de Sinaloa, Colegio de Sinaloa, México D.F., 417-425.

[4] Gurrola-López, G.H. (2000) Evaluación de los cambios en la cobertura vegetal y uso de suelo del sistema Lagunar Bahía Santa María, Angostura y Navolato, Sinaloa: Con aplicación de percepción remota. Tesis de maestría. Centro de Investigación en Alimentación y Desarrollo A.C.

[5] Fernández, G. and Lank, B.D. (2008) Effects of Habitat Loss on Shorebirds during the Nom Breeding Season: Current Knowledge and Suggestions for Action. Ornitología Neotropical, 19, 633-640.

[6] Tinajero, R. and Rodríguez, E.R. (2012) Efecto de la fragmentación del matorral desértico sobre la aguililla cola roja y el cernícalo americano en Baja California Sur, México. Acta Zoológica Mexicana, 28, 427-446.

[7] Pimm, S., Raven, P., Peterson, A., Sekercioglu, C.H. and Ehrlich, P.R. (2006) Human Impacts on the Rates of Recent, Present and Future Bird Extinctions. The National Academy of Sciences, 103, 10941-10946. https://doi.org/10.1073/pnas.0604181103

[8] Sarkar, S., Pressey, L.R., Faith, D.P., Margules, C.R., Fuller, T., Stoms, D.M., Moffett, A., Wilson, K.A., Williams, K.J., Williams, P.H. and Andelman, S. (2006) Biodiversity Conservation Planning Tools: Present Status and Challenges for the Future. Annual Review of Environment and Resources, 31, 123-159. https://doi.org/10.1146/annurev.energy.31.042606.085844

[9] Margules, C.R. and Pressey, R.L. (2000) Systematic Conservation Planning. Nature, 405, 
243-253. https://doi.org/10.1038/35012251

[10] Koleff, P., Tambutti, M., March, I.J., Esquivel, R., Cantú, C. and Lira-Noriega, A. (2009) Identificación de prioridades y análisis de vacíos y omisiones en la conservación de la biodiversidad de México. In: Capital Natural de México, Vol. II: Estado de conservación y tendencias de cambio, CONABIO, México, 651-718.

[11] Telleria, J.L. (2013) Pérdida de biodiversidad. Causas y consecuencias de la desaparición de las especies. Memorias de la Real Sociedad Española de Historia Natural, 10, 13-25.

[12] Singh, S.J. (2002) The Biodiversity Crisis: A Multifaceted Review. Current Science, 82, 638647.

[13] Escobar, E. and Maass, M. (2008) Diversidad de procesos funcionales en los ecosistemas. In: Capital Natural de México, Vol. 1: Conocimiento Actual de la Biodiversidad, Conabio, México, 161-189.

[14] López-Medellín, X., Navarro-Sigüenza, A.G. and Bocco, G. (2011) Human Population, Economic Activities, and Wild Bird Conservation in Mexico: Factors Influencing Their Relationships at Two Different Geopolitical Scales. Revista Mexicana de Biodiversidad, 82, 1267-1278.

[15] Navarro, S.A., Rebón, G.M., Gordillo, M.A., Townsend, P.A., Berlanga, G.H. and Sánchez, G.L. (2014) Biodiversidad de aves en México. Revista Mexicana de Biodiversidad, 85, 476495. https://doi.org/10.7550/rmb.41882

[16] Howell, S.N.G. and Webb, S. (1995) A Guide to the Birds of México and Northern Central America. Oxford Universidad Press, Oxford, $851 \mathrm{p}$.

[17] CONABIO (2016) Red de conocimiento sobre las aves de México. http://avesmx.conabio.gob.mx/EspeciesRegion.html\#ESTADO_25

[18] Contreras-Espinosa, F. and Castañeda-López, O. (2003) Los ecosistemas estuarinos del estado de Sinaloa. In: Cifuentes, L.J. and Gaxiola-López, J., Eds., Atlas de los Ecosistemas de Sinaloa, Colegio de Sinaloa, México, 175-196.

[19] Serrano, D., Ramírez-Félix, E. and Valle-Levinson, A. (2013) Tidal Hydrodynamics in a Two-Inlet Coastal Lagoon in the Gulf of California. Continental Shelf Research, 63, 1-12. https://doi.org/10.1016/j.csr.2013.04.038

[20] Engilis Jr., A., Oring, L.W., Carrera, E., Nelson, J.W. and Martinez-López, A. (1988) Shorebird Surveys in Ensenada Pabellones End Bahía Santa Maria, Sinaloa, México: Critical Winter Habitats for Pacific Flyway Shorebird. Wilson Bulletin, 110, 332-341.

[21] Vega-Picos, X., González-Bernal, M.A. and Muñoz del Viejo, A. (2006) Potential New Ramsar Sites in Northwest México: Strategic Importance for Migratory Waterbirds and Threats to Conservation. In: Boere, G.C., Galbraitn, C.A. and Stroud, D.A., Eds., Waterbirds around the World, the Stationary Office, Edinburgh.

[22] Villalba, A., Robadue, D. and Toscano, A. (2004) Estrategias para la conservación y desarrollo en Bahía de Santa María, México.

[23] Ralph, C.J., Geupel, G.R., Pyle, P., Martin, T.E. and DeSante, D.F. (1996). Handbook of Field Methods for Monitoring Landbirds. Gen. Tech. Rep. PSW-GTR-144, Pacific Southwest Research Station, Forest Service, US Department of Agriculture, Albany, 41 p.

[24] Molina, D., Torres-Guerrero, J. and Avelarde-Gómez, M.L. (2012) Riqueza de aves del Área Natural Protegida Estero El Salado, Puerto Vallarta, Jalisco, México. Huitzil, 13, 22-38.

[25] Peterson, T.R. and Chalif, E.L. (1989) Guía de campo Aves de México. Editorial Purrua, México, $458 \mathrm{p}$.

[26] Kaufman, K. (2005) Guía de campo a las aves de Norteamérica. Hillstar Editions LC, Sin- 
gapore, $391 \mathrm{p}$.

[27] Sibley, D.A. (2004) The Sibley Guide to Birds of Western North America. Alfred A Knopf Inc., New York, $471 \mathrm{p}$.

[28] Dunn, L.J. and Alderfer, J. (2008) Field Guide to the Birds of Western North America. National Geographic, Washington DC, 447 p.

[29] Zarate-Ovando, M.B., Palacio, E. and Reyes-Bonilla, H. (2008) Estructura de la comunidad y asociación de las aves acuáticas con la heterogeneidad espacial del complejo lagunar Bahía Magdalena-Almejas, Baja California Sur, México. Rev. Revista de Biología Tropical, 56, 371-389.

[30] Sánchez-Bon, M., Fernández, G., Escobedo, U.D., Torres, T.J. and Cid-Becerra, J.A. (2010) Composición espacial y temporal de la avifauna de las islas de barrera del complejo lagunar San Ignacio-Navachiste-Macapule, Sinaloa, México. Ciencias Marinas, 36, 355-370. https://doi.org/10.7773/cm.v36i4.1683

[31] Kasprzyk, M.J. and Harrington, B.A. (1989) Manual de campo para el estudio de las aves playeras. Playeras RHRPA, CICESE-MBO, Ensenada.

[32] Howes, J. and Bakewell, D. (1989) Shorebirds Studies Manual. Asian Wetland Bureau Publication No. 55, Kuala Lumpur, 362 p.

[33] American Ornithologists' Union (1998) Check-List of North American Birds. Séptima Edición, Washington DC, $829 \mathrm{p}$.

[34] Chesser, R.T., Banks, R.C., Cicero, C., Dunn, J.L., Kratter, A.W., Lovette, I.J., Navarro, S.A., Rasmussen, P.C., Remsen, J.V., Rising, J.D., Stotz, D.F. and Winker, K. (2014) Fifty-Fifth Supplement to the American Ornithogists' Union. Check-List of North American Birds. The Auk: Ornithological Advances, 131, CSi-CSxv.

[35] Secretaría de Medio Ambiente y Recursos Naturales (SEMARNAT) (2010) Norma Oficial Mexicana NOM-059-SEMARNAT-2010, Protección ambiental-Especies nativas de México de flora y fauna silvestres-Categorías de riesgo y especificaciones para su inclusión, exclusión o cambio-Lista de especies en riesgo. Diario Oficial de la Federación, México.

[36] Unión Internacional para la Conservación de la Naturaleza (2012) Categorías y Criterios de la Lista Roja de la UICN: Versión 3.1. Segunda Edición, Gland, Suiza y Cambridge, Reino Unido, UICN, $34 \mathrm{p}$.

[37] BirdLife International (2006) Conservando las Aves Migratorias Neotropicales en los Andes Tropicales. BirdLife International y US Fish and Wildlife Service, Quito, 105 p.

[38] Bojorges-Baños, J.C. (2006) Riqueza de especies de aves: propuestas metodológicas para su evaluación y estimación. Ciencia y Mar, 30, 59-64.

[39] Moreno, C.E. (2001) Métodos para medir la biodiversidad. M\&T-Manuales y Tesis SEA, Vol. 1, Zaragoza, 84 p.

[40] CONANP (2012) Estudio Previo Justificativo para el establecimiento del área natural protegida con la categoría de Área de Protección de Flora y Fauna Bahía de Santa María, en el Estado de Sinaloa. México, 142 p.

[41] Martínez-López, A. (2003) Fauna Silvestre. In: Cifuentes, L.J. and Gaxiola-López, J., Eds., Atlas de los ecosistemas de Sinaloa, Colegio de Sinaloa, México, 357-367.

[42] Leal, S.A., Fonseca, P.J. and Castillo, G.J.A. (2009) Noteworthy Bird Record from Central Sinaloa, México. Hiutzil, 10, 63-65.

[43] Carrascal, L.M. and Palomino D. (2005) Preferencias de hábitat, densidad y diversidad de las comunidades de aves en Tenerife (Islas Canarias). Animal Biodiversity and Conservation, 28, 101-119. 
Submit or recommend next manuscript to OALib Journal and we will provide best service for you:

- Publication frequency: Monthly

- 9 subject areas of science, technology and medicine

- Fair and rigorous peer-review system

- Fast publication process

- Article promotion in various social networking sites (LinkedIn, Facebook, Twitter, etc.)

- Maximum dissemination of your research work

Submit Your Paper Online: Click Here to Submit

Or Contact service@oalib.com 